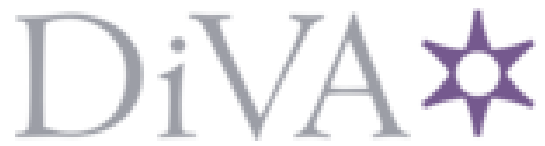

http://www.diva-portal.org

This is the published version of a paper published in Applied Physics Letters.

Citation for the original published paper (version of record):

Zahedinejad, M., Mazraati, H., Fulara, H., Yue, J., Jiang, S. et al. (2018)

CMOS compatible $\mathrm{W} / \mathrm{CoFeB} / \mathrm{MgO}$ spin Hall nano-oscillators with wide frequency tunability

Applied Physics Letters, 112(13): 132404

https://doi.org/10.1063/1.5022049

Access to the published version may require subscription.

N.B. When citing this work, cite the original published paper.

Permanent link to this version:

http://urn.kb.se/resolve?urn=urn:nbn:se:kth:diva-226793 


\section{CMOS compatible W/CoFeB/MgO spin Hall nano-oscillators with wide frequency tunability}

M. Zahedinejad, H. Mazraati, H. Fulara, J. Yue, S. Jiang, A. A. Awad, and J. Åkerman

Citation: Appl. Phys. Lett. 112, 132404 (2018); doi: 10.1063/1.5022049

View online: https://doi.org/10.1063/1.5022049

View Table of Contents: http://aip.scitation.org/toc/apl/112/13

Published by the American Institute of Physics

\section{Articles you may be interested in}

Spin-orbit torques in high-resistivity-W/CoFeB/MgO

Applied Physics Letters 112, 192408 (2018); 10.1063/1.5027855

Size dependence of the spin-orbit torque induced magnetic reversal in $\mathrm{W} / \mathrm{CoFeB} / \mathrm{MgO}$ nanostructures Applied Physics Letters 112, 142410 (2018); 10.1063/1.5022824

Influence of $\mathrm{MgO}$ barrier quality on spin-transfer torque in magnetic tunnel junctions

Applied Physics Letters 112, 022406 (2018); 10.1063/1.5005893

Spin-orbit torque-induced switching in ferrimagnetic alloys: Experiments and modeling

Applied Physics Letters 112, 062401 (2018); 10.1063/1.5017738

3D multilevel spin transfer torque devices

Applied Physics Letters 112, 112402 (2018); 10.1063/1.5021336

Direct writing of room temperature and zero field skyrmion lattices by a scanning local magnetic field Applied Physics Letters 112, 132405 (2018); 10.1063/1.5021172

\section{Conference Proceedings}




\title{
CMOS compatible W/CoFeB/MgO spin Hall nano-oscillators with wide frequency tunability
}

\author{
M. Zahedinejad, ${ }^{1}$ H. Mazraati, ${ }^{2,3}$ H. Fulara, ${ }^{1}$ J. Yue, ${ }^{1}$ S. Jiang, ${ }^{3}$ A. A. Awad, ${ }^{1}$ \\ and J. Ákerman ${ }^{1,2,3, a)}$ \\ ${ }^{1}$ Department of Physics, University of Gothenburg, 41296 Gothenburg, Sweden \\ ${ }^{2}$ NanOsc AB, 16440 Kista, Sweden \\ ${ }^{3}$ Department of Applied Physics, School of Engineering Sciences, KTH Royal Institute of Technology, \\ Electrum 229, SE-16440 Kista, Sweden
}

(Received 10 January 2018; accepted 17 March 2018; published online 29 March 2018)

\begin{abstract}
We demonstrate low-operational-current $\mathrm{W} / \mathrm{Co}_{20} \mathrm{Fe}_{60} \mathrm{~B}_{20} / \mathrm{MgO}$ spin Hall nano-oscillators (SHNOs) on highly resistive silicon (HiR-Si) substrates. Thanks to a record high spin Hall angle of the $\beta$-phase $\mathrm{W}\left(\theta_{S H}=-0.53\right)$, a very low threshold current density of $3.3 \times 10^{7} \mathrm{~A} / \mathrm{cm}^{2}$ can be achieved. Together with their very wide frequency tunability $(7-28 \mathrm{GHz})$, promoted by a moderate perpendicular magnetic anisotropy, $\mathrm{HiR}-\mathrm{Si} / \mathrm{W} / \mathrm{CoFeB}$ based $\mathrm{SHNOs}$ are potential candidates for wide-band microwave signal generation. Their CMOS compatibility offers a promising route towards the integration of spintronic microwave devices with other on-chip semiconductor microwave components. Published by AIP Publishing. https://doi.org/10.1063/1.5022049
\end{abstract}

The phenomenon of spin Hall effect ${ }^{1,2}$ has been widely exploited to generate a uniform pure transverse spin current density $\left(J_{s}\right)$ from a lateral charge current density $\left(J_{c}\right)$ using a non-magnetic metal (NM) with high spin-orbit interaction. The generated $J_{s}$ can diffuse into an adjacent ferromagnetic (FM) layer to apply spin orbit torque ${ }^{3}$ (SOT). Spin Hall nano-oscillators $^{4-8}$ (SHNOs) rely on SOT to overcome the local spin wave damping and sustain steady-state, current-, and field-tunable precession of the magnetization around an effective magnetic field. SHNOs have been demonstrated using different FM/NM combinations such as NiFe/Pt, 5,7,9,10 $\mathrm{NiFe} / \mathrm{W},{ }^{6} \mathrm{Co}_{40} \mathrm{Fe}_{40} \mathrm{~B}_{20} / \mathrm{Pt},{ }^{11}$ and $\mathrm{YIG} / \mathrm{Pt} .{ }^{12}$ Finding materials with a high charge-to-spin current conversion, quantified by the spin Hall angle $\left(\theta_{S H}=J_{S} / J_{c}\right)$, has been of particular interest, with $\mathrm{Pt}, \mathrm{Ta},{ }^{3} \mathrm{~W},{ }^{6} \mathrm{AuTa},{ }^{13}$ and $\mathrm{CuBi}^{14}$ being prominent examples.

For practical applications, it is crucial that the NM/FM stack can be grown with high quality on CMOS compatible substrates. Ideally, the substrate should also have a high thermal conductivity to dissipate the relatively large local heat generated by the SHNO and be inert to the NM layer at temperatures typically encountered during processing. For example, SHNO applications will likely require the use of $\mathrm{CoFeB} / \mathrm{MgO} / \mathrm{CoFeB}$ based magnetic tunnel junction (MTJ) stacks, which require an anneal step. The commonly used $\mathrm{Si} /$ $\mathrm{SiO}_{2}$ substrate suffers from poor thermal conductivity of $\mathrm{SiO}_{2}(1.3 \mathrm{~W} / \mathrm{mK})$. Therefore, other substrates were chosen for SHNOs, such as sapphire, ${ }^{5-7}$ which has a rather high thermal conductivity coefficient $(25 \mathrm{~W} / \mathrm{mK})$ and low microwave losses; however, sapphire is not a CMOS compatible substrate.

High resistivity $\mathrm{Si}$ could be an alternative substrate as it offers high thermal conductivity $(130 \mathrm{~W} / \mathrm{mK})$, both five times higher than sapphire and 100 times that of $\mathrm{Si} / \mathrm{SiO}_{2}$, while also exhibiting low microwave losses. ${ }^{15}$ However,

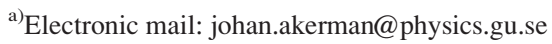

most of the typical metallic materials used in SHNOs, such as $\mathrm{Pt},{ }^{16} \mathrm{Pd},{ }^{17} \mathrm{Ni},{ }^{18} \mathrm{Cu}, \mathrm{Au}$, and their alloys, form lowresistivity $\mathrm{M}_{x} \mathrm{Si}_{y}$ silicides at elevated temperatures and hence would shunt the SHNO current, or inter-diffuse into the substrate, which is fatal for the CMOS process. Tungsten, on the other hand, can withstand very high temperatures up to $800^{\circ} \mathrm{C}$ without forming a silicide ${ }^{19}$ and is already widely used as a diffusion barrier in CMOS processes.

In this study, we therefore demonstrate SHNOs based on $\beta$-W/ $\mathrm{Co}_{20} \mathrm{Fe}_{60} \mathrm{~B}_{20} / \mathrm{MgO}$ stacks fabricated on high-resistivity silicon (HR-Si) substrates. Thanks to the very high spin Hall angle, $\theta_{S H}=-0.53$, these CMOS compatible SHNOs exhibit a very low auto-oscillation threshold current accompanied by a very wide current- and field-tunable microwave frequency. As STT-MRAM technology is pushed towards being embedded in fully depleted silicon on insulator (FD-SOI), which is widely used in $\mathrm{CMOS}^{20}$ and CMOS RF technology, ${ }^{21}$ our demonstration is also a proof-of-concept of how SHNOs can be embedded in FD-SOI.

The $\mathrm{W}(5) / \mathrm{Co}_{20} \mathrm{Fe}_{60} \mathrm{~B}_{20}(2) / \mathrm{MgO}(2)$ stack (thicknesses in $\mathrm{nm})$ was deposited using an AJA Orion-8 magnetron sputtering system working at a base pressure of $1 \times 10^{-8}$ mTorr, while the Argon pressure during sputtering was kept at 3 mTorr for all the layers. dc and rf sputtering was used for the depositions of metallic and insulting layers, respectively. As it is crucial to keep the $\mathrm{W}$ deposition rate low to obtain the $\beta$-phase with a high spin Hall angle, ${ }^{6,22}$ it was kept at $0.09 \AA / \mathrm{s}$; the same rate was used for $\mathrm{Co}_{20} \mathrm{Fe}_{60} \mathrm{~B}_{20}$, while that of the $\mathrm{MgO}$ layer was $0.04 \AA / s$. The stack was deposited on intrinsic HR-Si with $\rho_{\text {Si }}>10 \mathrm{k} \Omega \mathrm{cm}$.

To crystallize the $\mathrm{Co}_{20} \mathrm{Fe}_{60} \mathrm{~B}_{20}$ layer, the stack was annealed at $300{ }^{\circ} \mathrm{C}$ for $60 \mathrm{~min}$ at the system base pressure. The crystallization starts at the interface to $\mathrm{MgO}$, while $\mathrm{W}$ serves as a Boron getter ${ }^{23}$ to mediate the $\mathrm{Co}_{20} \mathrm{Fe}_{60} \mathrm{~B}_{20}$ crystallization. Annealing was followed by deposition of $40 \AA$ of $\mathrm{SiO}_{x}$ to protect the $\mathrm{MgO}$ layer from reacting with the ambient moisture. The $\beta$-phase $\mathrm{W}$ and $\mathrm{Co}_{20} \mathrm{Fe}_{60} \mathrm{~B}_{20}$ resistivities 
were measured to be $200 \mu \Omega \mathrm{cm}$ and $90 \mu \Omega \mathrm{cm}$, respectively. Obtaining a high resistivity $\mathrm{W}$ layer is a sign of having either mixed $\alpha+\beta$ or $\beta$-rich phase $\mathrm{W}$ as it has been studied extensively in the literature. ${ }^{6,22,24-26}$ For the sake of notation, We use the term $\beta$-phase for both cases in the rest of this manuscript.

In order to fabricate nano-constrictions, the sample surface was covered with negative electron resist followed by electron beam lithography (JEOL9300XS). Nano-constrictions of different widths were defined in $4 \mu \mathrm{m} \times 12 \mu \mathrm{m}$ mesas. We also made $6 \mu \mathrm{m} \times 18 \mu \mathrm{m}$ bars which were used to characterize the stack using spin-torque-induced ferromagnetic resonance (ST-FMR) measurements. The patterns were transferred to the stack by $\mathrm{Ar}$ ion beam etching using an Oxford Ionfab 300 Plus etcher. The negative resist was subsequently removed, and optical lift-off lithography was carried out to define ground-signal-ground (GSG) coplanar waveguides (CPW) of a thick $\mathrm{Cu}(950 \mathrm{~nm}) /$ $\mathrm{Au}(50 \mathrm{~nm})$ bilayer. To ensure a good electrical contact between the CPW and the SHNOs, the $\mathrm{MgO} / \mathrm{SiO}_{x}$ layers were removed in the CPW defined area by substrate plasma cleaning at a rf power of $40 \mathrm{~W}$ in our AJA Orion-8 sputtering machine right before $\mathrm{Cu} / \mathrm{Au}$ deposition.

Figure 1 shows a schematic of the SHNO device including the CPW. The inset shows the magnified view of the constituent layers and their thicknesses. A positive direct current is defined to flow from the signal pad to ground in the $y$-direction. $\phi$ and $\theta$ are the in-plane (IP) and out-of-plane (OOP) field angles, respectively.

We first investigated the magnetodynamic properties of the stack using the ST-FMR measurement on a $6 \times 18 \mu \mathrm{m}^{2}$ bar by applying a direct current (dc) and a $98.76 \mathrm{~Hz}$ modulated microwave current through the input port of a bias-tee at a fixed rf frequency and detecting the resulting voltage on its output port using a lock-in amplifier while sweeping the magnetic field $(0-0.2 \mathrm{~T})$. The voltage response from each sweep was fit to a sum of one symmetric and one antisymmetric Lorentzian sharing the same resonance field and linewidth. ${ }^{27}$ Figure 2(a) shows the resonance fields extracted at different microwave frequencies $(3-12 \mathrm{GHz})$ without any direct current. The field dependence is well described by a Kittel behavior ${ }^{28}$ with an effective magnetization of $M_{\text {eff }}$ $=0.71 \mathrm{~T}$ and a gyromagnetic ratio of $\gamma / 2 \pi=30.9 \mathrm{GHz} / \mathrm{T}$. It

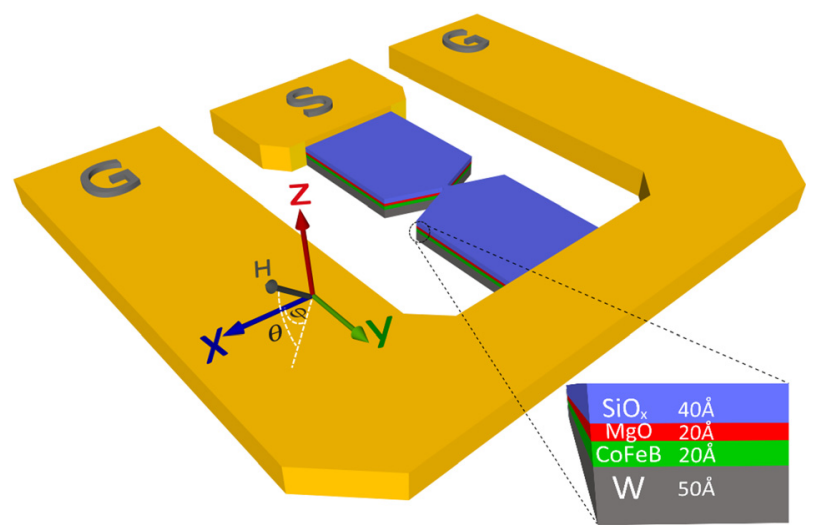

FIG. 1. Schematic of the SHNO device including the CPW. Positive direct current flows from the signal pad to ground in the $y$-direction. $\phi$ and $\theta$ are the in-plane (IP) and out-of-plane (OOP) field angles, respectively. The inset shows the layer order and thicknesses.
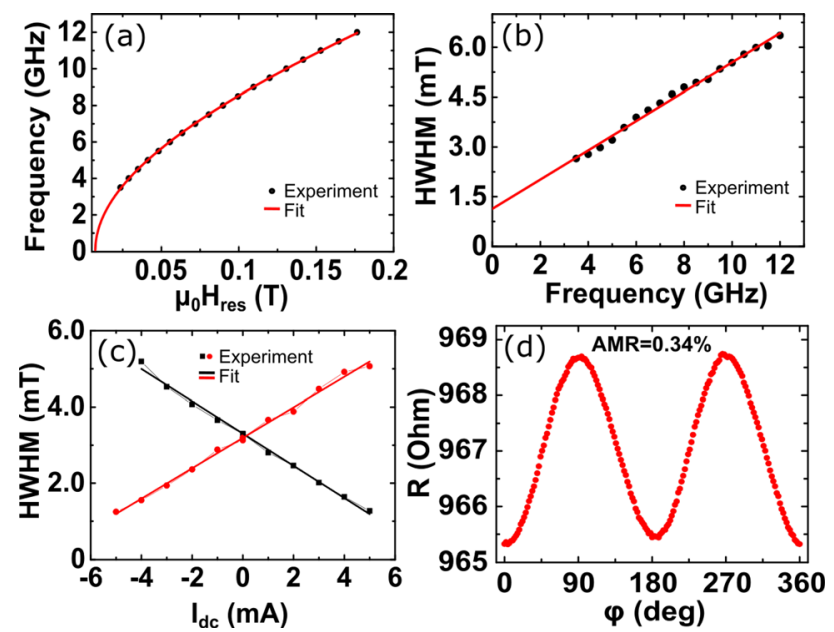

FIG. 2. (a) Resonance frequency vs. in-plane field from the ST-FMR measurement on a $6 \mu \mathrm{m}$-wide bar-shaped structure. (b) ST-FMR linewidth vs. frequency. (c) ST-FMR linewidth vs. current in an in-plane magnetic field along $\phi=30^{\circ}$ (black dots) and $210^{\circ}$ (red dots). (d) Obtained AMR for $\mathrm{w}=120 \mathrm{~nm}$ SHNO.

is interesting to compare $M_{\text {eff }}=0.71 \mathrm{~T}$ with $M_{S}=1.17 \mathrm{~T}$ measured using Alternating Gradient Magnetometry (AGM). According to equation $M_{\text {eff }}=M_{s}-H_{k}^{\perp}$. $H_{k}^{\perp}$, the significantly lower $M_{\text {eff }}$ indicates a moderate perpendicular magnetic anisotropy (PMA) field of $0.46 \mathrm{~T}^{29,30}$

Figure 2(b) shows the extracted linewidth for the same set of sweeps along with a linear fit ${ }^{28}$ yielding a low Gilbert damping of $\alpha=1.36 \times 10^{-2}$. Figure 2(c) shows the currentinduced linewidth changes in the ST-FMR spectra due to SOT, at a fixed frequency of $5 \mathrm{GHz}$. The linewidth has a linear current dependence with a negative (positive) slope for an IP field angle of $\phi=30^{\circ}\left(\phi=210^{\circ}\right)$ from which we can extract $\theta_{S H}=-0.53$, which is the highest reported $\theta_{S H}$ for W. ${ }^{22,24,25}$ Figure 2(d) shows an anisotropic magnetoresistance of $0.34 \%$ for a $120 \mathrm{~nm}$ wide nano-constriction. This is about an order of magnitude larger than the previously reported value of $0.026 \%$ for $\mathrm{CoFeB}^{11}$ and is related to the fact that our annealing step turns the initially amorphous $\mathrm{Co}_{20} \mathrm{Fe}_{60} \mathrm{~B}_{20}$ into a more poly-crystalline microstructure.

To investigate auto-oscillations, the lock-in amplifier was replaced with a low noise $+43 \mathrm{~dB}$ amplifier and a spectrum analyzer, and the generated microwave power spectral density (PSD) was recorded as a function of direct current and applied magnetic field. Figure 3(a) shows the current dependent PSD from a $w=120 \mathrm{~nm}$ nano-constriction measured in a constant $1 \mathrm{~T}$ field with $\phi=22^{\circ}$ and $\theta=60^{\circ}$. The non-monotonic current dependence-first red-shifted and then blue-shifted-is typical for a nano-constriction SHNO in a close-to-perpendicular magnetic field. ${ }^{5,31}$ The autooscillation region starts at the constriction edge (red-shift) and, as the current increases, moves to the center and expands to fill the constriction region (blue-shift). ${ }^{5}$ The linewidth [Fig. 3(b)] and integrated power [Fig. 3(c)] were obtained by Lorentzian fits to the PSD; the linewidth decreases and the power increases with increasing current. The maximum integrated power of about $8 \mathrm{pW}$ has been achieved at a low operational current of $0.9 \mathrm{~mA}$ which is considerably higher compared to previous reports on $\mathrm{NiFe/}$ $\mathrm{Pt}^{5,7}$ and $\mathrm{NiFe} / \mathrm{W}^{6}$ SHNOs. The high $\theta_{S H}$ reduces the auto- 


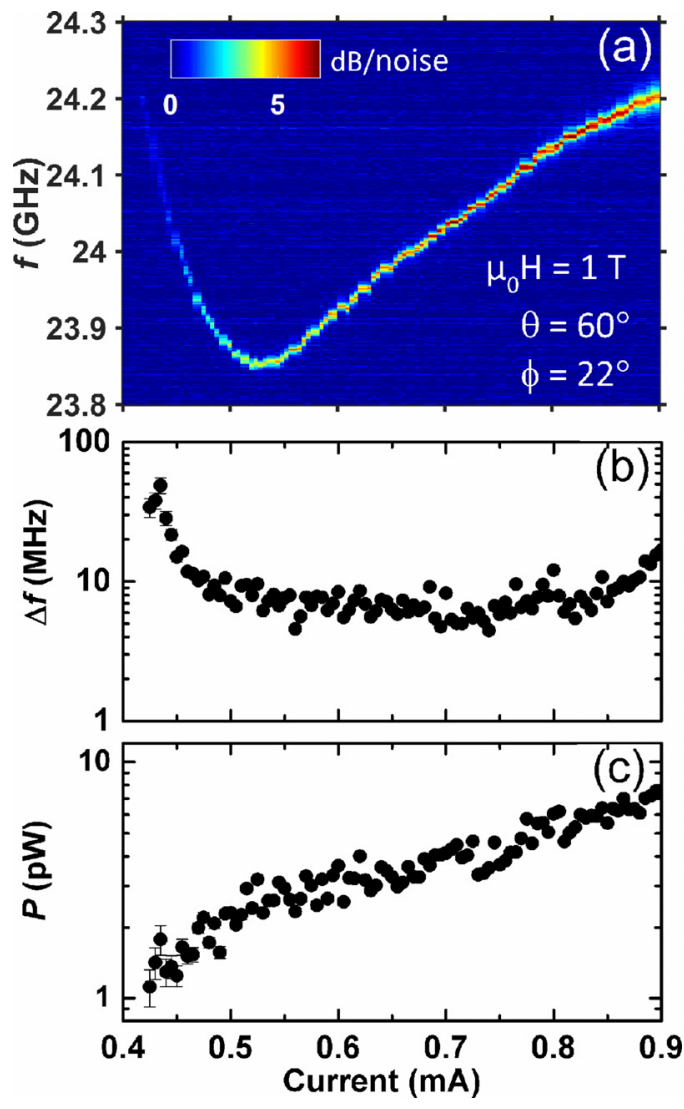

FIG. 3. (a) Power spectral density (PSD) vs. current for a $\mathrm{w}=120 \mathrm{~nm}$ SHNO in an applied magnetic field of $\mu_{0} H=1 \mathrm{~T}$ along $\phi=22^{\circ}$ and $\theta=60^{\circ}$. (b) Linewidth of the extracted auto-oscillation peaks. (c) Integrated power of the extracted individual peaks.

oscillation threshold current to about $I_{t h}=0.5 \mathrm{~mA}$ (determined as in Ref. 32), corresponding to a $4.4 \times 10^{7} \mathrm{~A} / \mathrm{cm}^{2}$ current density in the $\mathrm{W}$ layer, i.e., very close to previously reported values for $\mathrm{NiFe} / \mathrm{W}$ SHNOs in in-plane fields. ${ }^{6}$ Reducing the field magnitude to $0.8 \mathrm{~T}$, we extracted an even lower threshold of about $3.3 \times 10^{7} \mathrm{~A} / \mathrm{cm}^{2}$. It may be pointed out that a wider nano-constriction width offers an increased auto-oscillation mode volume which in turn reduces the linewidth of auto-oscillation peaks and enhances the peak power. However, lateral confinement of the nano-constriction, within the limit of reasonable yield of SHNO devices, results in a linear rise in resistance and an equivalent decrease in threshold current, thereby reducing the power consumption via the quadratic dependence on current. Therefore, an optimum nano-constriction width of $120 \mathrm{~nm}$ has been chosen in the present work to efficiently realize the conducive operational characteristics with lesser power consumption.

To examine the operational frequency range of our SHNOs, we investigated their auto-oscillation behavior as a function of OOP angle at a fixed field magnitude $\left(\mu_{0} H=1 \mathrm{~T}\right)$ and current $\left(I_{d c}=0.9 \mathrm{~mA}\right)$. Figure 4 displays the resulting auto-oscillations, which range from 12 to $28 \mathrm{GHz}$. As the OOP angle increases, the IP component of the magnetic field becomes smaller in magnitude and therefore shifts both the FMR frequency and the auto-oscillation operating frequency to lower values. It can be noted that our SHNOs are operative in a wide frequency range from $12 \mathrm{GHz}$ up to $28 \mathrm{GHz}$ by just tailoring the OOP angle. The inset in Fig. 4 further

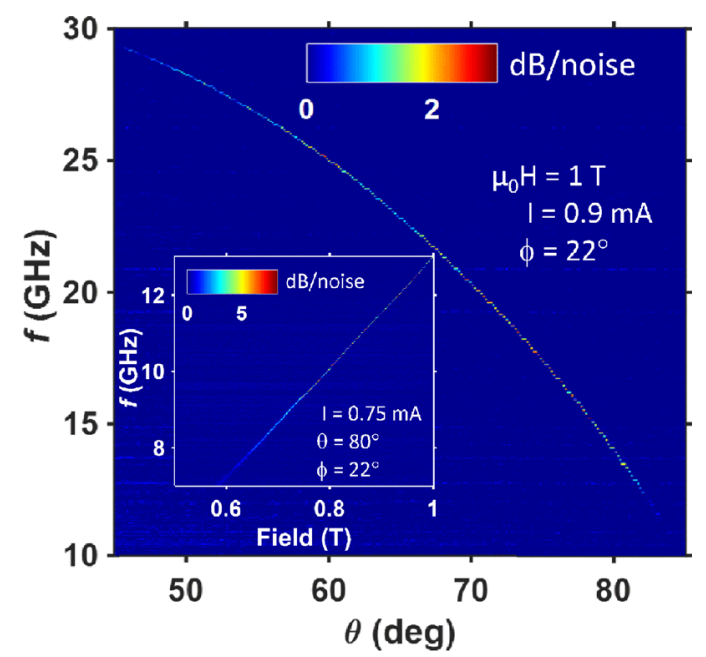

FIG. 4. Main panel: Out-of-plane angular dependence of the microwave precession frequency at fixed $\phi=22^{\circ}, I=0.9 \mathrm{~mA}$, and $\mu_{0} H=1 \mathrm{~T}$ for a $\mathrm{w}=120 \mathrm{~nm}$ SHNO. The inset displays the field dependence of microwave precession frequency for the same SHNO at fixed $I=0.75 \mathrm{~mA}$ along $\phi=22^{\circ}$ and $\theta=80^{\circ}$.

shows the field dependence of auto-oscillation frequency at a fixed OOP angle $\left(\theta=80^{\circ}\right)$ and dc-current $\left(I_{d c}=0.75 \mathrm{~mA}\right)$. The variation in the magnitude of the external magnetic field gives rise to the tilting of the internal magnetization angle with respect to the IP direction, which is manifested as a linear rise of the precessional frequency from $7 \mathrm{GHz}$ up to $13 \mathrm{GHz}$. It is noteworthy that an observable auto-oscillation signal clearly persists down to much lower field values than that suggested by $M_{S}$, which emphasizes the beneficial role of the moderate PMA in extending the field range. As a potential route to further reduce the required magnetic field to levels where integrated thin film permanent magnets would be sufficient, one could increase the PMA by thinning the $\mathrm{CoFeB}$ layer.

In conclusion, we have demonstrated CMOS compatible nano-constriction SHNOs based on $\mathrm{W} / \mathrm{Co}_{20} \mathrm{Fe}_{60} \mathrm{~B}_{20} / \mathrm{MgO}$ stacks grown on highly resistive $\mathrm{Si}$ substrates. Thanks to the record high spin Hall angle of the W layer $\left(\theta_{S H}=-0.53\right)$ and the moderate perpendicular magnetic anisotropy of the $\mathrm{Co}_{20} \mathrm{Fe}_{60} \mathrm{~B}_{20}$ layer, these SHNOs exhibit very low threshold currents and operate over a very wide frequency range, 7-28 GHz. Also, such devices could be further integrated on $\mathrm{Si}$ with other microwave components to offer more compact and tunable microwave devices used in RF CMOS communication systems.

This work was supported by the Swedish Foundation for Strategic Research (SSF), the Swedish Research Council (VR), and the Knut and Alice Wallenberg Foundation (KAW). This work was also supported by the European Research Council (ERC) under the European Community's Seventh Framework Programme (FP/2007-2013)/ERC Grant No. 307144 "MUSTANG".

${ }^{1}$ J. E. Hirsch, Phys. Rev. Lett. 83, 1834-1837 (1999).

${ }^{2}$ J. Sinova, S. O. Valenzuela, J. Wunderlich, C. H. Back, and T. Jungwirth, Rev. Mod. Phys. 87, 1213-1260 (2015).

${ }^{3}$ L. Liu, C.-F. Pai, Y. Li, H. Tseng, D. Ralph, and R. Buhrman, Science 336, 555-558 (2012). 
${ }^{4}$ L. Liu, C.-F. Pai, D. C. Ralph, and R. A. Buhrman, Phys. Rev. Lett. 109, 186602 (2012).

${ }^{5}$ A. Awad, P. Dürrenfeld, A. Houshang, M. Dvornik, E. Iacocca, R. Dumas, and J. Åkerman, Nat. Phys. 13, 292-299 (2016).

${ }^{6}$ H. Mazraati, S. Chung, A. Houshang, M. Dvornik, L. Piazza, F. Qejvanaj, S. Jiang, T. Q. Le, J. Weissenrieder, and J. Åkerman, Appl. Phys. Lett. 109, 242402 (2016).

${ }^{7}$ P. Dürrenfeld, A. A. Awad, A. Houshang, R. K. Dumas, and J. Åkerman, Nanoscale 9, 1285-1291 (2017).

${ }^{8}$ T. Chen, R. K. Dumas, A. Eklund, P. K. Muduli, A. Houshang, A. A. Awad, P. Dürrenfeld, B. G. Malm, A. Rusu, and J. Åkerman, Proc. IEEE 104, 1919-1945 (2016).

${ }^{9}$ V. E. Demidov, S. Urazhdin, H. Ulrichs, V. Tiberkevich, A. Slavin, D. Baither, G. Schmitz, and S. O. Demokritov, Nat. Mater. 11, 1028-1031 (2012).

${ }^{10}$ V. E. Demidov, S. Urazhdin, A. Zholud, A. V. Sadovnikov, and S. O. Demokritov, Appl. Phys. Lett. 105, 172410 (2014).

${ }^{11}$ M. Ranjbar, P. Dürrenfeld, M. Haidar, E. Iacocca, M. Balinskiy, T. Q. Le, M. Fazlali, A. Houshang, A. A. Awad, R. K. Dumas, and J. Åkerman, IEEE Magn. Lett. 5, 3000504 (2014).

${ }^{12}$ M. Collet, X. de Milly, O. d'Allivy Kelly, V. V. Naletov, R. Bernard, P. Bortolotti, J. Ben Youssef, V. E. Demidov, S. O. Demokritov, J. L. Prieto, M. Munoz, V. Cros, A. Anane, G. de Loubens, and O. Klein, Nat. Commun. 7, 10377 (2016).

${ }^{13}$ P. Laczkowski, Y. Fu, H. Yang, J.-C. Rojas-Sánchez, P. Noel, V. T. Pham, G. Zahnd, C. Deranlot, S. Collin, C. Bouard et al., Phys. Rev. B 96, 140405 (2017).

${ }^{14}$ Y. Niimi, Y. Kawanishi, D. H. Wei, C. Deranlot, H. X. Yang, M. Chshiev, T. Valet, A. Fert, and Y. Otani, Phys. Rev. Lett. 109, 156602 (2012).

${ }^{15}$ A. C. Reyes, S. M. El-Ghazaly, S. Dorn, M. Dydyk, D. K. Schroder, and H. Patterson, in 1996 Proceedings 46th Electronic Components and Technology Conference (IEEE, 1996), pp. 382-391.
${ }^{16}$ S. Cohen, P. Piacente, G. Gildenblat, and D. Brown, J. Appl. Phys. 53, 8856-8862 (1982).

${ }^{17}$ S. P. Murarka, Intermetallics 3, 173-186 (1995).

${ }^{18}$ J. Luo, Z. Qiu, C. Zha, Z. Zhang, D. Wu, J. Lu, J. Åkerman, M. Östling, L. Hultman, and S.-L. Zhang, Appl. Phys. Lett. 96, 031911 (2010).

${ }^{19}$ M. Siegal, W. Graham, and J. Santiago, J. Appl. Phys. 66, 6073-6076 (1989).

${ }^{20}$ B. Doris, B. DeSalvo, K. Cheng, P. Morin, and M. Vinet, Solid-State Electron. 117, 37-59 (2016).

${ }^{21}$ G. G. Shahidi, IBM J. Res. Dev. 46, 121-131 (2002).

${ }^{22}$ C. Zhang, S. Fukami, K. Watanabe, A. Ohkawara, S. DuttaGupta, H. Sato, F. Matsukura, and H. Ohno, Appl. Phys. Lett. 109, 192405 (2016).

${ }^{23}$ S.-E. Lee, T.-H. Shim, and J.-G. Park, NPG Asia Mater. 8, e324 (2016).

${ }^{24}$ S. Mondal, S. Choudhury, N. Jha, A. Ganguly, J. Sinha, and A. Barman, Phys. Rev. B 96, 054414 (2017).

${ }^{25}$ K.-U. Demasius, T. Phung, W. Zhang, B. P. Hughes, S.-H. Yang, A. Kellock, W. Han, A. Pushp, and S. S. P. Parkin, Nat. Commun. 7, 10644 (2016).

${ }^{26}$ C.-F. Pai, L. Liu, Y. Li, H. Tseng, D. Ralph, and R. Buhrman, Appl. Phys. Lett. 101, 122404 (2012).

${ }^{27}$ L. Liu, T. Moriyama, D. C. Ralph, and R. A. Buhrman, Phys. Rev. Lett. 106, 036601 (2011).

${ }^{28}$ C. Kittel, Phys. Rev. 73, 155 (1948).

${ }^{29}$ X. Liu, W. Zhang, M. J. Carter, and G. Xiao, J. Appl. Phys. 110, 033910 (2011).

${ }^{30}$ S. Peng, M. Wang, H. Yang, L. Zeng, J. Nan, J. Zhou, Y. Zhang, A. Hallal, M. Chshiev, K. L. Wang et al., Sci. Rep. 5, 18173 (2015).

${ }^{31}$ M. Dvornik, A. A. Awad, and J. Åkerman, Phys. Rev. Appl. 9, 014017 (2018).

${ }^{32}$ V. Tiberkevich, A. Slavin, and J.-V. Kim, Appl. Phys. Lett. 91, 192506 (2007). 